\title{
FREE PRODUCTS OF ORDERED SEMIGROUPS
}

\author{
R. E. JOHNSON
}

Several years ago A. A. Vinogradov [1] proved that the free product of two ordered groups is orderable. In this note we give a simplified version of his proof, which also holds for semigroups.

A semigroup $S$ is said to be ordered iff it has a transitive linear ordering $<$ such that whenever $a<b$ then $a c<b c$ and $c a<c b$ for all $c \in S$. Clearly, each ordered semigroup is cancellative. Since each ordered semigroup can be imbedded in an ordered semigroup with identity (letting $1<a$ iff $a<a^{2}$ ), we can assume without loss of generality that each semigroup considered below has an identity element.

Given two ordered semigroups $S_{1}$ and $S_{2}$, we can form their cartesian product $S_{1} S_{2}$ and their free product $S_{1} * S_{2}$. The orders in $S_{1}$ and $S_{2}$ can easily be extended to $S_{1} S_{2}$, perhaps in many different ways. For example, if $a_{i}, b_{i} \in S_{i}$, we can define $a_{1} a_{2}>b_{1} b_{2}$ in $S_{1} S_{2}$ iff either $a_{1}>b_{1}$ or $a_{1}=b_{1}$ and $a_{2}>b_{2}$. The aim of this paper is to prove the following result.

Theorem. The free product $S_{1} * S_{2}$ of ordered semigroups $S_{1}$ and $S_{2}$ is orderable. In fact, for each ordering of $S_{1} S_{2}$, there exists an ordering of $S_{1} * S_{2}$ such that the natural homomorphism $S_{1} * S_{2} \rightarrow S_{1} S_{2}$ is order preserving.

This theorem is proved by imbedding $S_{1} * S_{2}$ in an ordered semigroup of infinite-dimensional matrices as follows. Let $R=Q\left(S_{1} S_{2}\right)$ be the semigroup algebra of $S_{1} S_{2}$ over the rational field $Q$. For any given order of $S_{1} S_{2}$, we can order $R$ in the familiar way: if $s_{i} \in S_{1} S_{2}$ and $a_{i} \in Q$ with $s_{1}<s_{2}<\cdots<s_{n}$ and all $a_{i} \neq 0$, let $0<\sum_{i=1}^{n} a_{i} s_{i}$ iff $0<a_{n}$. Clearly, $R$ is an integral domain with unity, and $S_{1} S_{2} \subset R$.

Consider now the set $T$ of all upper triangular $\omega \times \omega$ matrices over $R$ whose diagonal elements are in $S_{1} S_{2}$. In terms of matrix units $\left\{e_{i j} \mid i, j=1,2, \cdots\right\}, T$ consists of all matrices of the form

$$
A=\sum_{i, j=1}^{\infty} a_{i j} e_{i j}, \quad a_{i i} \in S_{1} S_{2}, a_{i j}=0 \text { if } i>j .
$$

Each $A=\left(a_{i j}\right)$ can be expressed in terms of its diagonals:

$$
A=\sum_{k=0}^{\infty} A_{k}, \quad A_{k}=\sum_{i=1}^{\infty} a_{i+k} e_{i+k} .
$$

Received by the editors May 17, 1966 and, in revised form, March 2, 1967. 
If $B=\sum B_{k} \in T$, then the $k$ th diagonal of $A B$ is

$$
(A B)_{k}=\sum_{i=0}^{k} A_{i} B_{k-i} .
$$

It is evident that $T$ is a multiplicative semigroup with identity $I$.

The semigroup $T$ can be ordered relative to diagonals. In the first place, if $A_{k}=\sum a_{i+k} e_{i+k}$ and $B_{k}=\sum b_{i+k} e_{i+k}$ are $k$ th diagonal matrices, let $A_{k}<B_{k}$ iff there exists an integer $r$ such that $a_{i+k}$ $=b_{i+k}$ for all $i<r$ and $a_{r+k}<b_{r+k}$. Then for any $A=\sum A_{k}$ and $B=\sum B_{k}$ in $T$, let $A<B$ iff there exists an integer $k$ such that $A_{i}=B_{i}$ for all $i<k$ and $A_{k}<B_{k}$. It is easily shown that $<$ is a transitive linear ordering in $T$.

To complete the proof that $T$ is an ordered semigroup, let $A=\sum A_{k}$, $B=\sum B_{k}$, and $C=\sum C_{k}$ be in $T$, with $A<B$. Hence, $A_{i}=B_{i}$ if $i<k$ and $A_{k}<B_{k}$ for some integer $k$. Since $(A C)_{i}=(B C)_{i}$ and $A_{i} C_{k-i}$ $=B_{i} C_{k-i}$ if $i<k$, and $A_{k} C_{0}<B_{k} C_{0}$, evidently $(A C)_{k}<(B C)_{k}$. Therefore, $A C<B C$. Similarly, we can show $C A<C B$. Hence, $T$ is an ordered semigroup.

We imbed $S_{1} S_{2}$ in $T$ by identifying each $x \in S_{1} S_{2}$ with the diagona matrix

$$
D_{x}=(x-1) D_{0}+I=\operatorname{diag}(1, x, 1, x, \cdots),
$$

where

$$
D_{0}=\sum_{i=1}^{\infty} e_{2 i 2 i}
$$

Clearly the order in $T$ is an extension of the order in $S_{1} S_{2}$.

The injection of $S_{1} * S_{2}$ in $T$ is accomplished as follows. Let the diagonal matrices $A_{1}$ and $B_{1}$ be defined by

$$
A_{1}=\sum_{i=1}^{\infty} e_{2 i-12 i}, \quad B_{1}=\sum_{i=1}^{\infty} e_{2 i 2 i+1} .
$$

Then $A_{1}^{2}=B_{1}^{2}=0$, whereas $A_{1} B_{1}$ and $B_{1} A_{1}$ are nonnilpotent diagonal matrices. Also, $A_{1} D_{0}=A_{1}, D_{0} A_{1}=0, B_{1} D_{0}=0$, and $D_{0} B_{1}=B_{1}$. For each $x \in S_{1} S_{2}$, let us define

$$
\begin{aligned}
& H_{x}=\left(I+A_{1}\right)^{-1} D_{x}\left(I+A_{1}\right)=D_{x}+(1-x) A_{1}, \\
& K_{x}=\left(I-B_{1}\right)^{-1} D_{x}\left(I-B_{1}\right)=D_{x}+(1-x) B_{1} .
\end{aligned}
$$

Now we define the mapping $S_{1} * S_{2}^{\phi} T$ as follows:

$$
\phi(\cdots x y \cdots)=\cdots H_{x} K_{y} \cdots, \quad x \in S_{1}, y \in S_{2} .
$$


Since $\phi(x)=H_{x}$ and $\phi(y)=K_{y}$ for $x \in S_{1}, y \in S_{2}$, evidently $\phi \mid S_{1}$ and $\phi \mid S_{2}$ are homomorphisms. Hence, $\phi$ itself is a homomorphism.

To prove that $\phi$ is actually a monomorphism, let $x=a_{1} a_{2} \cdots a_{n}$ be an element of $S_{1} * S_{2}$ of length $n$. Thus, $a_{i} \in S_{1}$ if $i$ is odd, $a_{i} \in S_{2}$ if $i$ is even (or vice versa), and all $a_{i} \neq 1$. The last nonzero diagonal of $\phi(x)=H_{a_{1}} K_{a_{2}} \cdots$ is the $n$th diagonal as given below:

$$
\prod_{i=1}^{n}\left(1-a_{i}\right) \sum_{j=1}^{\infty} e_{2 j-1} 2 j+n-1 .
$$

If $y=b_{1} \cdots b_{m}$ is an element of $S_{1} * S_{2}$ of length $m$, then the $m$ th diagonal of $\phi(y)$ is

or

$$
\prod_{i=1}^{m}\left(1-b_{i}\right) \sum_{j=1}^{\infty} e_{2 j-1} 2 j+m-1
$$

$$
\prod_{i=1}^{m}\left(1-b_{i}\right) \sum_{j=1}^{\infty} e_{2 j}{ }_{2 j+m},
$$

depending on whether $b_{1} \in S_{1}$ or $b_{1} \in S_{2}$. Before $\phi(x)$ can equal $\phi(y)$, evidently $m$ must equal $n$ and $a_{1}, b_{1} \in S_{1}$ (or $a_{1}, b_{1} \in S_{2}$ ).

Let us assume that for all elements of $S_{1} * S_{2}$ of length less than $n$, whenever $\phi(x)=\phi(y)$ then $x=y$. Assume that $x=a_{1} \cdots a_{n}$, $y=b_{1} \cdots b_{n}$ have length $n$, that $a_{1}, b_{1} \in S_{1}$, and that $\phi(x)=\phi(y)$. Since the $n$th diagonals of $\phi(x)$ and $\phi(y)$ are equal,

$$
\prod_{i=1}^{n}\left(1-a_{i}\right)=\prod_{i=1}^{n}\left(1-b_{i}\right)
$$

The $(n-1)$ th diagonal of $\phi(x)$ is

$$
D_{a_{1}}\left(1-a_{2}\right) B_{1}\left(1-a_{3}\right) A_{1} \cdots+\left(1-a_{1}\right) A_{1}\left(1-a_{2}\right) B_{1} \cdots D_{a_{n}} .
$$

Thus, the even-row coordinates of the $(n-1)$ th diagonal of $\phi(x)$ are all equal to $a_{1}\left(1-a_{2}\right) \cdots\left(1-a_{n}\right)$. Since $\phi(x)=\phi(y)$, we must have

$$
a_{1} \prod_{i=2}^{n}\left(1-a_{i}\right)=b_{1} \prod_{i=2}^{n}\left(1-b_{i}\right) .
$$

From (1) and (2) and the fact that $R$ is an integral domain, we get $a_{1}=b_{1}$. If $x^{\prime}=a_{2} \cdots a_{n}$ and $y^{\prime}=b_{2} \cdots b_{n}$, then $\phi\left(a_{1}\right) \phi\left(x^{\prime}\right)=\phi\left(b_{1}\right) \phi\left(y^{\prime}\right)$, and hence $\phi\left(x^{\prime}\right)=\phi\left(y^{\prime}\right)$. Therefore, by the induction assumption, $x^{\prime}=y^{\prime}$ and $x=y$. An analogous argument holds if $a_{1}, b_{1} \in S_{2}$. We conclude that $\phi$ is a monomorphism. Therefore, the ordering in $T$ induces an ordering in $S_{1} * S_{2}$. 
If $x \in S_{1} * S_{2}$ maps in to $x^{\prime} \in S_{1} S_{2}$ under the natural homomorphism $S_{1} * S_{2} \rightarrow S_{1} S_{2}$, then $\phi(x)$ has main diagonal $D_{x^{\prime}}$. Since the ordering in $T$ is an extension of the ordering in $S_{1} S_{2}$, evidently the natural homomorphism $S_{1} * S_{2} \rightarrow S_{1} S_{2}$ is order preserving. This completes the proof of the theorem.

Once we have shown how to order the free product of two ordered semigroups, we can order the free product of any well-ordered set of ordered semigroups by induction.

A feature of the construction in the proof above is that it allows us to compute the relationship between elements in any specific case. As a simple example, let $S_{1}=\left\{x_{1}^{a} \mid a \in Q^{+}\right\}$and $S_{2}=\left\{x_{2}^{a} \mid a \in Q\right\}$, where $x_{1}$ and $x_{2}$ are indeterminates, $Q$ is the additive group of rationals, and $Q^{+}=\{a \in Q \mid a \geqq 0\}$. We can order $S_{1} S_{2}$ by total degrees as follows: $x_{1}^{a_{1}} x_{2}^{a_{2}}>x_{1}^{b_{1}} x_{2}^{b_{2}}$ iff $a_{1}+a_{2}>b_{1}+b_{2}$ or $a_{1}+a_{2}=b_{1}+b_{2}$ and $a_{1}>b_{1}$. If, for example, $y=x_{2}^{-1} x_{1} x_{2}$, then $\phi(y)=D_{x_{1}}+\left(x_{2}-x_{1} x_{2}\right) A_{1}$ $+\left(1-x_{1}-x_{2}+x_{1} x_{2}\right) B_{1}+$ higher diagonals. Since $x_{2}-x_{1} x_{2}<0$, we have $y<x_{1}$. Similarly, if $z=x_{2} x_{1} x_{2}^{-1}$ then $\phi(z)=D_{x_{1}}+\left(x_{2}^{-1}-x_{1} x_{2}^{-1}\right) A_{1}$ $+\left(1-x_{1}-x_{2}^{-1}-x_{1} x_{2}^{-1}\right) B_{1}+$ higher diagonals and $z<x_{1}$. Also, $x_{2}^{-1}$ $-x_{1} x_{2}^{-1}>x_{2}-x_{1} x_{2}$ and therefore $z>y$.

\section{REFERENCE}

1. A. A. Vinogradov, On the free product of ordered groups, Mat. Sb. 25 (1949), 163-168. (Russian)

UNiversity of Montana 